\title{
Quantity VS Quality: Logical-Philosophical and Cognitive Bases of Interaction
}

\author{
Elena $V$. Fedyaeva ${ }^{1, *}$ \\ ${ }^{1}$ Novosibirsk State Technical University Humanities Department 20 K. Marksa Pr. 630072 Novosibirsk, Russia
}

\begin{abstract}
The article discusses some interaction aspects of basic universal categories of quantity and quality. Within the logical-philosophical approach, the categories of quantity and quality reflect the objective aspects of existence and the reality objects relationship. This explains the significant correlation between these categories and language: different knowledge formats about the quantitative and qualitative side of being find their linguistic embodiment. Studying these categories interaction from the standpoint of human cognitive activity within anthropocentric linguistics and cognitive-discursive paradigm reveals some new potential for analysis which implies the possibility of qualitative characteristics interpretation of language units with quantitative meaning. The conclusions are: 1) both quality and quantity reflect the objects properties and characteristics, thus showing a close connection which enables the interpretation of quality via quantity; 2) ontologically motivated association of quantity and quality signifies that a language unit contains both explicit (quantitative) and potentially inferred implicit (qualitative) characteristics; 3) QUANTITY as a modus or evaluative concept is the result of the quantitative aspect of being comprehension and is capable to profile the qualitative characteristics of the reality objects; 4) the phenomenon of inference, acting as a 'bridge' between quantity and quality is a cognitive basis for the quantitative interpretation of quality.
\end{abstract}

\section{Logical-philosophical interpretation of quantity and quality}

Quantity and quality are extremely significant ontological categories: they are universal categories of existence and, consequently, are among the fundamental categories of philosophy. The categories of quantity and quality are present in all social processes, being the basic categories of dialectics. This accounts for their significance in the theoretical understanding of the human society development, in the fundamental qualitative transformations taking place in various aspects of public life, and in the dynamism of the global social process.

Plotinus noted that "the multiplicity of the thinking elements yields the category of quantity and number, and their diversity gives the category of quality. These principles, or the highest categories, give rise to the subsequent ones, as well as to all types and kinds of things" [1, p. 16]. Since an object is always the unity of certain quality and quantity (the qualitative quantity and quantitative value of quality), these categories reflect the components that synthesize the results of sensory cognition of the reality diversity in human mind.

The category of quality acts as an initial stage of human cognitive activity. The logical category of quality was formed in the process of labor activity when humans started creating and using some objects and realized their inherent properties.
The concepts of property and state belong to the quality category as particular forms of quality manifestation. According to Aristotle, property is regarded as a quality which is stable and can hardly be subject to change. Owing to the stability of property as a kind of quality, an individual can distinguish one sort of things from another. The attributes indicating the object's state are, in Aristotle's view, the second meaning of quality. State, in Aristotle's view, is the manifestation of quality which is less stable in comparison with the property, prone to changes and able to disappear. States are the types of qualities that are easily amenable to change, such as heat and cold, sickness and health, and all those similar states [2].

Thus, the formation of the conceptual quality category precedes the formation of conceptual quantity category as the knowledge of the objects quantitative certainty is possible only after these objects are differentiated from the reality as qualitatively defined (on the basis of their common quality).

Further, in the process of establishing the objects quantitative certainty, human mind is distracted from these objects qualitative certainty considering them as qualitatively homogeneous items.

It was Aristotle who presented the significant characteristics of the quantity category underlying its modern interpretation: 'Quantum' means that which is divisible into two or more constituent parts of which each is by nature a 'one' and a 'this'. A quantum is a 
plurality if it is numerable, a magnitude if it is a measurable. 'Plurality' means that which is divisible potentially into non-continuous parts, 'magnitude' that which is divisible into continuous parts [2]. It is clear from this definition that at the earliest stages of the quantity category development it was perceived as an ambiguous and complex one. The definition by Aristotle contains the indication of a certain distinction within the quantity category concerning the discrete, i.e. counted quantity, and continuous (non-discrete) i.e. measurable quantity. This division is caused by the variety of quantitatively characterized real world objects and phenomena.

\section{Quantity and quality in everyday human life}

It is especially significant that quantity can be thought of 'per se' only if it is completely abstracted from the qualitative features of the counted items. Only in this case the category of quantity is independent from other categories and is mainly intended to express exact mathematical forms of quantity reflecting scientific concepts of the high abstraction order, i.e. numbers.

However, the characteristic properties of numbers, which are formed in the human mind and imprinted in symbolic numerical code, indicate that a non-scientific interpretation of a number is often characterized by a "living" image with certain attributes. For instance, the qualitative character of numbers is directly perceived in the conditional division of numbers on the so-called 'unhappy' and 'happy'. Thus, the number thirteen in Western cultures is often perceived as 'unhappy'. According to V. F. Hopper, this fact allows for multiple explanations, rather than a single one. One explanation relates to the need of correlating the early lunar calendars with the solar year, which required adding the thirteenth 'superfluous' month. The intercalated thirteen month was regarded as discordant and unlucky. The omnipresent 13 of the lunar cycle made the number fearsome. At the same time, the number may have become popularly associated with the diabolical art. In Faust's Miraculous Art and Book of Marvels, or the Black Raven, 13 are said to compose the Infernal Hierarchy. In Britain, 13 became associated with witchcraft. Whether for the same reason or because the inclusion of a leader with any group of 12 makes a thirteen, as seems to have been the case in Druidic ceremony, a witches' coven was ordinarily composed of 13. The famous 'unlucky 13' and especially the ' 13 at table' is indisputably connected with the Last Supper [3, p. 131-132].

One of the forms of number functioning in matter is the quantity which has logical-philosophical nature and occupies a significant place in the conceptual image of the world. The quantity receives qualitative specification through the objects or phenomena which are exposed to counting. Being a higher-order abstraction, the number contains no evaluation and in this aspect opposes quantity which, as it is shown by the data of psychological research, refers to the semantic zone of the
Gestalt "WORLD EVALUATION" by an individual [4, p. 197].

Speaking of quantity, we always mean the quantity of something. The quantity receives qualitative specification through the objects or phenomena which are exposed to counting. G. Frege noted that "in their scientific use in mathematics, number words are proper names like 'Sirius' or 'Africa'. What a proper name designates is an object. Mathematics therefore regards numbers as objects, not as properties. It uses number words substantially, not predicatively. This distinguishes the scientific use of number words in mathematics from their unscientific use in daily life, where the use of 'four horses' is similar to that of 'big horses', as if 'four' gave us a property of an individual horse like 'big'; and yet, while in the case of big horses every individual is big, in the case of four horses not every individual is four $[5, \mathrm{p}$. 178]. It is evident that in everyday life the number is directly connected with the world of objects, being one of the most abstract concepts departing from material reality.

The close interconnection of quantity and quality and their importance are revealed, for example, in various conventional forms of quantity in the human society. This fact is explained by the aspiration to standardize or to find a common measuring basis for the multitude of subjective points of view regarding certain qualitative characteristics. For example, certain conventional forms of the quality functioning in the field of medicine are designed to fix the basic vital indicators (such as body temperature, heart rate, respiratory rate, blood pressure) by which the state of an individual is evaluated.

The correlations between quantity and quality implying quality evaluation in quantitative terms are of great importance for the everyday life of the society. This is also reflected in traditional educational systems of results evaluation (for example, in Russia 5 is 'excellent', 4 is 'good', 3 is 'satisfactory') and in different measurement systems, scales, ratings and classifications. The widely known and universal, for example, is the socalled "star" classification system characterizing hotels, restaurants, food, drinks in terms of quantity. For example, the rating developed by Michelin company, which is considered to be a bible of the gastronomic industry, is intended to evaluate, first of all, the quality of the cuisine: the number of stars (maximum number is three) next to the name of the restaurant always shows that the atmosphere, service, interior and price niche of the restaurant are secondary to the quality of the dishes served there. The number of stars on some kinds of alcohol products usually testifies to their age, and therefore to qualitative characteristics, as in the examples:

1) Renaldo's was one of the country's premier spots with a Michelin star to its name and a twenty-year reputation for fabulous food and wonderful service [1, p. 46].

2) Roger's favourite drink was a 'zonker', and his evenings at home would consist of two zonkers before dinner, then wine with. The zonker itself might be a champagne cocktail - a finger of three-star cognac, a lump of sugar, a single drop of bitters and a tumblerful 
of very cold biscuity champagne or it might be a dry martini or a straightforward whisky with ice and soda [2, p. 347].

It becomes obvious that by reflecting the basic patterns of reality, the categories of quantity and quality are closely interconnected testifying to the close interconnection of all phenomena in general. In addition, these examples indicate the relationship of these categories to the category of evaluation, i.e. the formation of an opinion about quality and quantity becomes possible due to the evaluation activities of human mind.

Thus, considering these categories as a system as well as tracing the systematic relationship of these categories to the category of evaluation is an important feature of understanding the quantitative and qualitative aspects of being.

\section{Quantity and quality: cognitive approach}

In the process of mental activity, an individual basically evaluates the reality thus realizing the evaluative attitude to the world in the process of cognition. Within the modern framework of anthropocentric approach to language, an individual is accepted to possess a special internal vision of the world. Researchers also argue that perception, conceptualization, categorization of the world by an individual include the evaluative or interpretative component. Interpretation, being an integral characteristic of human mind, can be understood as a peculiar 'anthropocentric view' at the state of reality.

According to N.N. Boldyrev, interpretation is "the kind of cognitive activity, the process and the results of human understanding and explaining the world and human role in this world, the process and the result of the world representation, which is based on the one hand, on the collective perceptions of the world and, on the other hand, on a individual experience of interaction with it" [6, p. 28].

The interpretation process would not be possible without the cognitive process of inference: thought operation which results in the ability to go beyond literal/verbatim meaning of language units, to see more content behind the considered language forms and to determine what they imply and what can be inferred using everyday experience and everyday cognition" [7, p. 33-34].

The appeal to the problem of inference is caused by the desire to understand the nature of human reasoning in general. Being one of the basic universal cognitive processes, inferential capacity is often associated with the guesses that an individual makes on the basis of the experience and intuition or on the use of different types of knowledge.

Aristotle is considered to be the first who wrote on the process of inference having formulated a set of principles governing the syllogism. The modern research in different fields (cognitive computing and artificial intelligence, experimental psychology, neuroscience, linguistics) aims at investigating various mechanisms, developing diverse models of inferential human information processing: ground cognition theories (L. W. Barsalou [8]), mental models theory (P. N. JohnsonLaird [9]), situation models (T. A. van Dijk, W. Kintsch [10], R. A. Zwaan, G.A. Radvansky [11]), etc.

It is of crucial importance that the realization of the interpreting function in the language would not be possible without a special system of modus or evaluative categories, which provide the possibility of speaker's different interpretations of a conceptual content and the formation of new separate meanings on the basis of this interpretation. One of the distinguishing characteristics of this type of category is their inference, i.e. the need for the inferred knowledge to generate the necessary meaning: the elements of evaluative categories can imply a number of thought operations without revealing the exact content of the thought object [12, p. 170].

In this regard, it is quite reasonable to address the problem of the inference phenomenon in the process of quantitative interpretation of quality. QUANTITY as a modus type concept (underlying the category of quantity) is connected with inclusion of an individual ability to interpret the objects and phenomena in terms of quantitative attributes (characteristics). The result of this reflection is the language units, representing conceptual knowledge, derived from the mental activity dealing with the quantitative side of being, i.e. the quantitative evaluation of objects and events of the reality, representing the attitude of the speaker to the evaluated quantity. At the same time this evaluation is in compliance with the communicative rules adopted in a linguocultural community. Consequently, as the modus or evaluative type of concept, QUANTITY represents the result of human comprehension of the quantitative aspect of being. Furthermore, it demonstrates the ability to profile the qualitative characteristics of the real world objects and phenomena.

It is of further interest that both quality and quantity reflect qualitative and quantitative characteristics or properties, thus providing the possibility of quality interpretation via quantity.

The ontologically reasoned connection of quantity and quality can signify that a language unit possesses both explicit quantitative and potentially inferred implicit qualitative characteristics. It can be assumed that the structures of knowledge representation, which are the basis for inferred qualitative knowledge, are activated in the process of quantitative parameters perception.

The interpretation depends on the defined field and stipulates the salient characteristics. For instance, the interpretation of quantitative parameters within the thematic conceptual domain HUMAN BEING can be connected with comprehension of human properties, as in the examples:

3) At the television studios, the security guard on reception was hauling a big bag of fan mail with red envelopes spilling out of the top, through the inner security doors to the offices. 'Don't know what they see in him,' muttered the guard, panting. 'He's got a face like a robber's dog. Five foot two is all' [1, p. 99].

4) Wim had been six feet three by the time he reached fifteen [3, p.18]. 
The given examples illustrate that the modus or evaluation function of quantity is realized, for example, by numerical markers, which become the basis for understanding human height as one of the anthropometric characteristics. The formation of the meanings 'rather short' (3) and 'rather tall' (4) is actualized on the basis of inference. The source of these meaning is the stereotypic knowledge of average height within the relevant measurement system for a given culture. It is of special interest that despite the concept QUANTITY being the basic universal concept of human mind inherent in any culture, the language representation of QUANTITY is culture specific. This can be exemplified by the use of different measurement systems by different linguocultures. Thus, the English system of measures used in the UK, the USA and other countries is independent and distinct from others.

This kind of specificity can also be applied to the size range of clothing:

5) 'He's married to Ingrid Fitzgerald, for heaven's sake - she's only a size 12' [1, p.13].

6) Star could never resist pottery, but she hadn't been into the lingerie department, despite Lena explaining about their biggest seller: a range made by a former home economics teacher from Dublin who was fed up with trying to get comfortable suck-it-all-in underwear for women over size 18, and had designed her own range [1, p.13].

The interpretation of human physical characteristics in examples (5) and (6) is a schematic representation of experience in accordance with the collective system of norms based on a certain scale and adopted within the given culture, which leads to the formation of meanings 'rather slender' and 'rather corpulent' respectively. In addition, example (6) is an example of rationally reflected free from emotions evaluation, which is consistent with the communicative conventions of UNDERSTATEMENT.

The empirical material analysis shows that within the thematic conceptual domain of HUMAN BEING the quantitative parameters can serve as an interpretation instrument of a wide repertoire of qualitative characteristics, including various states:

7) She looked tiny next to him, but he knew her well enough now to know that her spirit was ten feet tall $[4$, p. 384].

8) 'It was horrendous, I felt about two inches tall,' Charlie admitted [1, p. 376].

The linguistic interpretation of the emotional and psychological state of a person in such precise quantitative terms, presented in the above examples, is likely to reflect the specificity of the English-speaking culture, mentality and communication ethics, and, consequently, the specificity of the English language. Stated by J. Locke, the principles of adherence to the facts, the accuracy of the thought formulation, the clear separation of scientific knowledge and opinion had a great influence on the formation of the collective consciousness not only of the British, but also of other English-speaking (American, Australian, Canadian) cultures that inherited these principles. The intensive development of scientific thought contributed to the formation of the ideal image of English as a language which must meet the need to express the thought as accurately as possible and free of emotions [13, p. 141].

\section{Conclusion}

Summing up, it should be noted that the result of quantitative interpretation of quality is the establishment of conceptual relations which have a predominantly logical character. They are the consequence of the close relations between the categories of quantity and quality motivated by the world ontology and the evaluative activity of human mind. The expression of quality in terms of quantity testifies to the correlation of conceptual domains caused by the continuous character of human mind and the diffusive boundaries between categories.

The study of the QUANTITY potential to interpret QUALITY emphasizes the special role of an individual as an interpreter of reality. Thus, there is a certain contribution to the solution of the "central for the whole cognitive science question, namely the question of what is added by an individual into the immediate experience and what is not deducible from this experience by induction. But the fact that imagination, fantasy, intuition, memory and other cognitive abilities always contribute to the development and comprehension of the world seems indisputable" [14, p. 82]. Cognitive approach to language learning provides the possibility of finding the knowledge structures behind the language forms, as well as the mechanisms that motivate the language representation of knowledge. It also grants the possibility of considering the already known phenomena, namely quantity and quality, from a new perspective.

\section{References}

1. Plotin, Selected works (M: RM, 1994)

2. Aristotle, Metaphysics-Aristotle. Nuvision Publications (2005).

3. V.F. Hopper, Medieval number symbolism: its sources, meaning, and influence on thought and expression (N.Y., Mineola: Dover Publications Inc., 2000)

4. Yu. N. Karaulov, Language steps And images of the world (M., 2000)

5. G. Frege, Philosophical and mathematical correspondence (Chicago: The University of Chicago Press, 1980)

6. N.N. Boldyrev, Language. Culture. Transfer. Communication (M.: Tezaurus, 2015)

7. E.S. Kubryakova, V.Z. Dem'yankov, Yu.G. Pankrats, L.G. Luzina, Abridged Dictionary of Cognitive Terms (M.: MGU im. M.V. Lomonosova, 1997).

8. L.W. Barsalou, Philosophical Transactions of the Royal Society B: Biological Science 364, 12811289 (2009)

9. P. N. Johnson-Laird, Cognitive Science 4, 71-115 (1980) 
10. T. A. van Dijk, W. Kintsch Strategies of Discourse Comprehension (New York: Academic Press) (1983)

11. R. A. Zwaan, G. A. Radvansky, Psychological Bulletin 123(2),162-185 (1998)

12. N.N. Boldyrev, Cognitive Nature of Language: Collected Essays (M.-Berlin : Direkt-Media, 2016)

13. L.A. Kozlova, Ethnocultural Potential of a Language Grammatical Structure and its Realization in the Grammar of the Speaker (Barnaul: AltGPA, 2009)

14. E.S. Kubryakova Language and Knowledge: On the Way of Acquiring Knowledge about Language: Parts of Speech in Cognitive Framework. The Role of Language in the World Cognition (M.: Languages of Slavic culture, 2004)

\section{Sources}

1. C. Kelly, Once in a Lifetime. (Lnd. : Harper, 2012)

2. S.A. Faulks, Week in December (Lnd.: Vintage, 2010)

3. D. Steel, Dating Games. (Lnd.: Corgi Books, 2004)

4. D. Steel, Bungalow 2 (Lnd.: Corgi Books, 2008) 